Check for updates

Cite this: RSC Adv., 2018, 8, 25021

Received 10th May 2018

Accepted 2nd July 2018

DOI: 10.1039/c8ra03980a

rsc.li/rsc-advances

\section{Experimental study of the protective effect of mesosilica-supported 5-hydroxymethylfurfural on UV-induced aging of human dermal fibroblasts}

\begin{abstract}
Xueming Niu, $\mathbb{D}+^{\mathrm{a}}$ Zhenyu Wang, $\hat{\dagger}^{\mathrm{d}}$ Long Zhang, ${ }^{\mathrm{b}}$ Yunyun Quan ${ }^{\star b c}$ and Kun Wei ${ }^{\star a b}$
The drug 5-HMF (5-hydroxymethylfurfural, $\mathrm{C}_{6} \mathrm{H}_{6} \mathrm{O}_{3}$ ) is extensively studied for its antioxidative and antiinflammatory properties. However, its unstable properties and biotoxicity restrict its use in skin care products and therapy. The present study was aimed at evaluating the potential of three-dimensional dendritic mesoporous silica nanospheres (3D-dendritic MSNs) as a topical carrier system for 5-HMF delivery. The encapsulation of the carrier also enhances the stability of the drug. Based on the results of Brunauer-Emmet-Teller (BET) analysis, Fourier transform infrared spectroscopy (FTIR), transmission electron microscopy (TEM), thermogravimetric analysis (TGA), dynamic light scattering (DLS), and UV-vis diffuse reflectance spectroscopy, drug delivery systems were successfully fabricated and the loading capacity (LC\%) and entrapment efficiency (EE\%) were also assessed. In vitro cell tests revealed the outstanding biocompatibility and inoxidizability of 3D-dendritic MSNs. There is no effect on the antioxidant properties of the drug. Therefore, mesoporous silica can be combined with 5-HMF and used as potential antioxidant medicine in cosmetic applications.
\end{abstract}

\section{Introduction}

Skin aging is a complex biological phenomenon consisting of two components: intrinsic and extrinsic aging. ${ }^{1}$ The process of intrinsic or natural aging occurs not only in the skin but also in all other human tissues. It is an inevitable consequence of physiological and genetic changes. ${ }^{1,2}$ Extrinsic aging is caused by cumulative exposure to external stimuli, including ultraviolet radiation, environmental toxins, and life style, which induce DNA alterations and damage the skin. ${ }^{3}$

With the rapid economic development, environmental problems have become increasingly serious, such as the hole in the ozone layer. For this reason, sunlight is the most harmful external component threatening the skin. Ultraviolet B (UVB) exposure generally results in increased generation of reactive oxygen species (ROS) within the cell, which can subsequently lead to oxidative stress and produce photodamage in proteins and other macromolecules in the skin. ${ }^{4}$

\footnotetext{
${ }^{a}$ School of Biological Science and Engineering, South China University of Technology, Guangzhou 510640, China. E-mail: weikun@scut.edu.cn; Tel: +8613902848267 ${ }^{b}$ Wenzhou Institute of Biomaterials and Engineering, CAS, Wenzhou, Zhejiang 325011, China

${ }^{c}$ Engineering Research Center of Clinical Functional Materials and Diagnosis \& Treatment Devices of Zhejiang Province, Wenzhou Institute of Biomaterials and Engineering, CAS, Wenzhou, Zhejiang 325011, China

${ }^{d}$ Department of Environmental Engineering, College of Ecology, Lishui University, Lishui 323000, China

$\dagger$ These authors contributed equally to this work.
}

Overexposure can cause a series of diseases, such as malignant melanoma of the skin, skin squamous cell carcinoma, and basal cell carcinoma of the skin. ${ }^{5,6}$ Currently, skin cancers are the most common cancer among all other organ malignancies.

5-HMF (5-hydroxymethylfurfural, $\mathrm{C}_{6} \mathrm{H}_{6} \mathrm{O}_{3}$ ) ${ }^{7}$ is a five-carbonring aromatic aldehyde that exists naturally in dried fruits, fruit juices, and flavoring agents. It is also an important fine chemical raw material and a common intermediate product that can be obtained through the degradation of hexoses and the Maillard reaction. ${ }^{8}$

Despite the previous reviews on the dangers of 5-HMF, there has been an accepted theory concerning the biological activity and good antioxidant effects of 5-HMF in recent years. ${ }^{8}$ Many researchers have studied its properties in different cells. Kim et al. ${ }^{9}$ reported that 5-HMF had an anti-inflammatory effect on Human Umbilical Vein Endothelial Cells (HUVEC). Li et al. ${ }^{10}$ isolated and characterized 5-HMF by nuclear magnetic resonance (NMR) from Laurencia undulata and evaluated the antioxidant using various assays at the molecular and RAW264.7 cellular levels. The antioxygenation processes including the scavenging of free-radical and cellular ROS were also studies. Zhao et al. ${ }^{7}$ studied the antioxidative and antiproliferative activities of 5-HMF on A375 cells. They found that it can reduce the levels of ROS and malondialdehyde (MDA) and increase the levels of superoxide dismutase (SOD), catalase (CAT), and glutathione peroxide (GPx).

However, very few studies address the antioxidant effects of 5-HMF on skin cells. As a novel natural antioxidant, the study of 5-HMF in skin cells will be of great significance, and can be used 
to reduce the incidence of skin diseases. However, the instability of 5-HMF was an obstacle in the drug promotion process. Thus, a feasible scheme is urgently needed to solve this problem.

To overcome these shortcomings, the use of mesoporous silica nanospheres (MSNs) was proposed. ${ }^{11,12}$ MSNs are novel, uniform, monodispersed, three-dimensional dendritic mesoporous silica nanospheres (3D-dendritic MSNs). ${ }^{13}$ The 3Ddendritic MSNs have hierarchical mesostructures with multigenerational, tunable center-radial and dendritic mesopore channels. ${ }^{14}$ More importantly, the 3D-dendritic MSNs have faster biodegradation rates in simulated body fluid with larger pore sizes. ${ }^{\mathbf{1 3 , 1 5 , 1 6}}$ MSNs have been investigated particularly as promising drug delivery platforms due to some of their outstanding virtues, such as large surface area, tunable pore size distributions, and the fact that it can be modified by different functional groups. ${ }^{17,18}$ Therefore, mesoporous silica nanospheres are widely used in drug transportation. ${ }^{\mathbf{1 9 , 2 0}}$

This paper describes how we designed and synthesized a 3Ddendritic mesoporous silica-based drug delivery system. The mesoporous silica nanospheres act as carriers for the unstable 5-HMF, and are called 5-HMF@MSNs. Nanospheres with smaller particle size, better dispersion, and larger specific surface area were obtained by adjusting the ratio of emulsifier and TEOS, the $\mathrm{pH}$ of the reaction solvent, and optimizing the reaction time. 3D-dendritic MSNs were prepared by the one-pot continuous interfacial growth method. GPx and matrix metalloproteinase (MMP-1) kits were used to prove the antioxidant effect. Bioactivities were evaluated to confirm the drug delivery system's cytotoxicity. Through a one-pot method, we can obtain nanospheres with good particle size uniformity.

It was concluded that 5-HMF had good antioxidation and proliferation effects on skin cells and can scavenge free radicals and enhance the activity of antioxidant enzymes. When 5-HMF is loaded into a carrier, it achieves better biocompatibility and oxidation resistance. 5-HMF@MSNs have great potential in cosmetics and medical diets.

\section{Experiments and methods}

\section{Materials}

Cetyltrimethylammonium chloride (CTAC) solution (25 wt\% in $\mathrm{H}_{2} \mathrm{O}$ ) and tetraethyl orthosilicate (TEOS), triethanolamine (TEA) were purchased from Sigma-Aldrich (UK), 1-octadecene, decahydronaphthalene and cyclohexane were purchased from Beyotime Biotechnology, human skin fibroblast (HSF) cells were purchased from KunMing Cell Bank and cultivated in Roswell Park Memorial Institute-1640 with 10\% fetal bovine serum (FBS) at $37{ }^{\circ} \mathrm{C}$ in a $95 \%$ air, $5 \% \mathrm{CO}_{2}$ atmosphere.

\section{Ultraviolet B irradiation and treatment}

Ultraviolet B treatment of the HSF cells was performed as follows. The HSFs were plated at a density of $8 \times 10^{3}$ cells per well in 96-well plates in $200 \mu \mathrm{L}$ of supplemented medium overnight. Then, the medium was removed. The cells were washed once with phosphate buffer saline (PBS) before adding

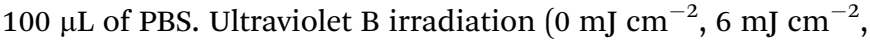
$12 \mathrm{~mJ} \mathrm{~cm}^{-2}, 18 \mathrm{~mJ} \mathrm{~cm}^{-2}, 24 \mathrm{~mJ} \mathrm{~cm}^{-2}, 30 \mathrm{~mJ} \mathrm{~cm}^{-2}$ ) was performed using a UV Irradiation System for Cultures (PHILIPS UVB 311NM). The emitted radiation was checked using a UVB radiometer with a sensor. After irradiation, the cells were incubated at $37{ }^{\circ} \mathrm{C}$ with FBS-free medium. Senescent HSFs were identified by stained SA- $\beta$-galactosidase with X-Gal (5-bromo-4chloro-3-indolyl $\beta$-D-galactopyranoside). We can detect the mitochondrial membrane potential in irradiated cells by the mitochondrial staining for JC-1 to detect the mitochondrial membrane potential.

\section{The antioxidant effect of 5-hydroxymethylfurfural for HSF}

We proved the enzyme activities of superoxide dismutase, and a large number of researchers have studied this activity. These experiments were performed according to the manufacturer's kit instructions. The superoxide dismutase activity level was determined by the nitroblue tetrazolium method, which used a tetrazolium salt to quantify the superoxide radicals generated by xanthine oxidase and hypoxanthine. Data were obtained by measuring the absorbance at $450 \mathrm{~nm}$ of SOD.

\section{Synthesis of 3D-dendritic MSNs}

By optimizing the experimental conditions, our novel method of synthesizing 3D-dendritic MSNs was performed. A total of $24 \mathrm{~mL}$ of cetyltrimethylammonium chloride (CTAC) and $0.18 \mathrm{~g}$ of triethanolamine (TEA) were dissolved in $36 \mathrm{~mL}$ of deionized water in a $250 \mathrm{~mL}$ conical flask at $60^{\circ} \mathrm{C}$ and stirred gently for $1 \mathrm{~h}$ to obtain the template solution. Then, $20 \mathrm{~mL}$ of $(10 \mathrm{v} / \mathrm{v} \%)$ TEOS in 1-octadecene was carefully added to the water-CTAC-TEA solution and kept at $60{ }^{\circ} \mathrm{C}$ in an oil bath under mechanical stirring for $12 \mathrm{~h}$. Then, the upper phase, 1-octadecene solution was completely removed and replaced with $10 \mathrm{v} / \mathrm{v} \%$ TEOS solution in decahydronaphthalene to keep the same reaction condition for another $12 \mathrm{~h}$ for the growth of the second generation. The upper oil layer was changed to a solution of $10 \mathrm{v} / \mathrm{v} \%$ TEOS in cyclohexane and the reaction conditions were maintained for $12 \mathrm{~h}$ for the last generation. The products were calcined in the muffle furnace at $500{ }^{\circ} \mathrm{C}$ for $300 \mathrm{~min}$ to remove the template.

\section{Characterization}

The 3D-dendritic MSNs were visualized using a JEM-2100F (Jeol Ltd, Japan) transmission electron microscope (TEM) and a Hitachi S-4800 (Hitachi Ltd, Japan) scanning electron microscope (SEM). Fourier transform infrared (FT-IR) spectra were recorded on a Tensor II (Bruker, Germany). The size distribution and zeta potential of the nanospheres was measured by dynamic light scattering (DLS) using a Zetasizer Nano ZS double-angle dynamic laser-scattering instrument (Malvern, UK). The mesoporous structures were confirmed by BrunauerEmmett-Teller (BET) analysis. The Thermo Gravimetric Analyzer (TGA) results confirmed the successful loading of the drug. The biological activity was determined using a UV-vis spectrophotometer (ThermoFisher, USA). 

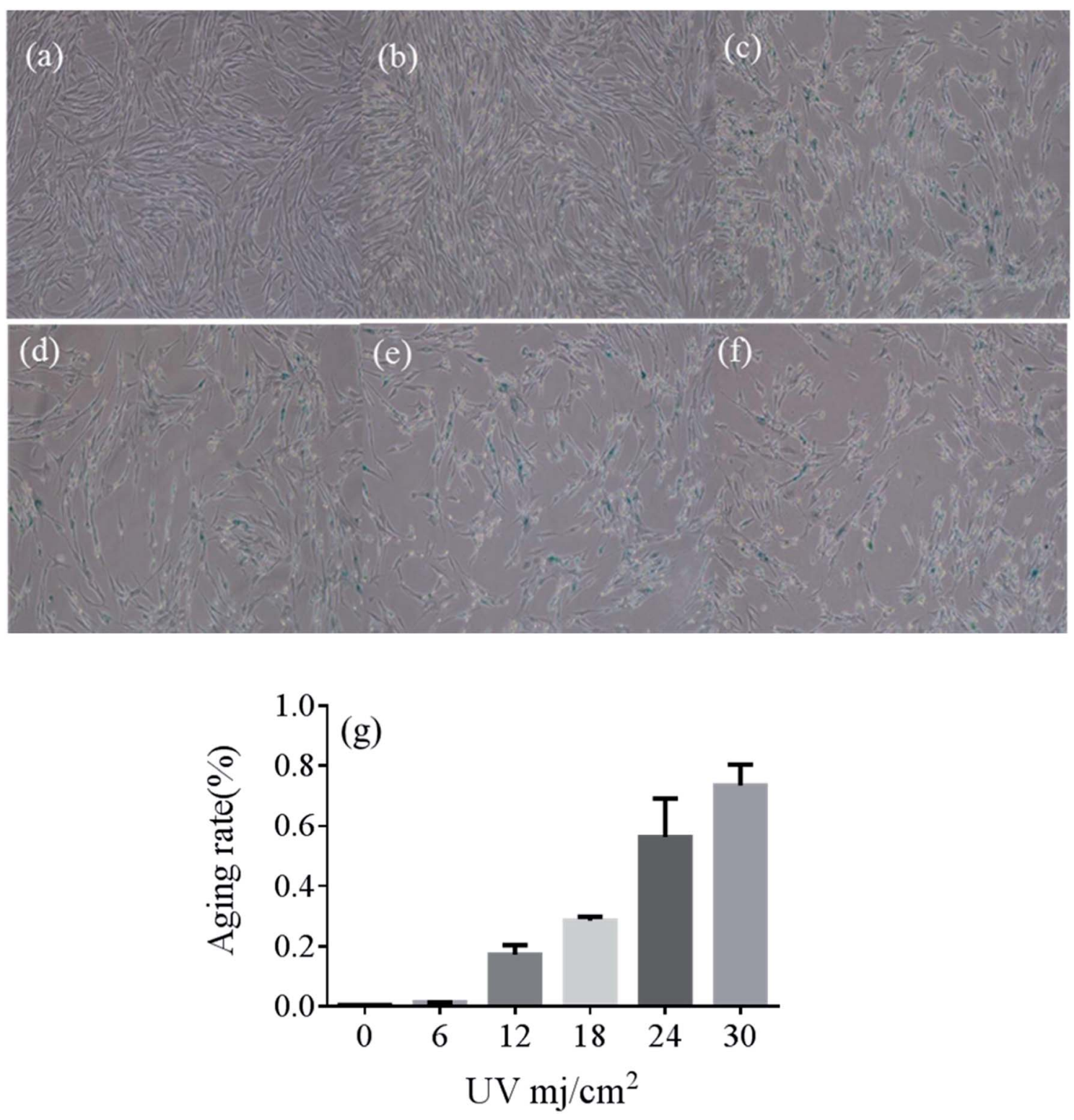

Fig. 1 (a) -(f) The staining of SA- $\beta$-galactosidase: (a) UVB- $0 \mathrm{~mJ} \mathrm{~cm}^{-2}$, (b) UVB-6 mJ cm $\mathrm{cm}^{-2}$, (c) UVB-12 mJ cm $\mathrm{cm}^{-2}$, (d) UVB-18 mJ cm ${ }^{-2}$, (e) UVB-24 $\mathrm{mJ} \mathrm{cm} \mathrm{cm}^{-2}$, (f) UVB-30 mJ cm${ }^{-2}$, and (g) the percentage of SA-b-gal-positive cells.

\section{Synthesis of 5-HMF-loaded MSNs (5-HMF@MSNs)}

Briefly, $10 \mathrm{mg}$ of 3D-dendritic MSNs were dispersed in $20 \mathrm{~mL}$ PBS containing $5 \mathrm{mg}$ of 5 -HMF by ultrasound treatment for $30 \mathrm{~min}$. The suspension was continuously stirred under vacuum process for $30 \mathrm{~min}$ to remove the air inside the mesopores. Then, $20 \mathrm{~mL} \mathrm{H}_{2} \mathrm{O}$ was added to the solution to promote recrystallization of the drugs inside the mesoporous channels. The reactor was shaken for $16 \mathrm{~h}$. Then, the drug-loaded silica nanospheres were collected by centrifugation $(10000 \mathrm{rpm}, 10$ min), and washed with ethanol several times.

\section{Loading capacity (LC\%) and entrapment efficiency (EE\%) of 5- HMF-loaded samples}

The standard concentration curve of 5-HMF was determined using absorbance values. The 5-HMF solution concentrations

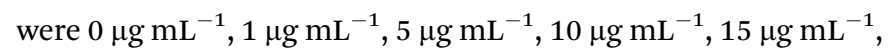
$20 \mu \mathrm{g} \mathrm{mL}{ }^{-1}, 25 \mu \mathrm{g} \mathrm{mL} L^{-1}$, and $80 \mu \mathrm{g} \mathrm{mL}{ }^{-1}$, respectively. The maximum absorption wavelength was determined with an ultraviolet visible spectrometer, and the absorbance value was measured at the wavelength $\left(\lambda_{\max }=298 \mathrm{~nm}\right)$. The standard curve was obtained:

$$
\begin{gathered}
Y=28.87 \times X-12.904 \\
R^{2}=0.9997
\end{gathered}
$$

where $Y$ is the absorbance and $X$ is the concentration $\left(\mu \mathrm{g} \mathrm{mL} L^{-1}\right)$.

The 5-HMF@MSNs supernatant was monitored at the wavenumber $298 \mathrm{~nm}$. The LC\% and EE\% were calculated using the following equations. The actual proportion of the 5-HMF on MSNs and the 5-HMF@ MSNs are defined as LC\% and EE\% 


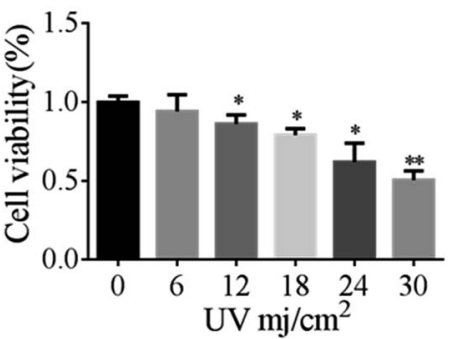

Fig. 2 Cytotoxicity at different doses of UVB irradiation.

respectively, after being washed in ethanol. Typically, LC\% and EE\% for different weight ratios of 5 -HMF : MSNs (0.5:1, $0.75: 1,1: 1,1: 2 \mathrm{w} / \mathrm{w})$ were investigated to achieve the optimal parameters of drug loading procedures:

$$
\begin{aligned}
& \mathrm{LC} \%=\frac{M_{\mathrm{t}} / M_{\mathrm{s}}}{M} \times 100 \% \\
& \mathrm{EE} \%=\frac{M_{\mathrm{t}} / M_{\mathrm{s}}}{M_{\mathrm{t}}} \times 100 \%
\end{aligned}
$$

where $M_{\mathrm{t}}, M_{\mathrm{s}}, M$ are the initial weight of 5-HMF, the weight of the drug in the supernatant, and the weight of the drug in the drug delivery system, respectively.

\section{In vitro 5-HMF release profile of 3D-dendritic MSNs}

The 5-HMF release properties of the drug delivery nanosystem and the degradation effect of the MSNs were investigated. 5HMF@MSNs and pure 5-HMF were placed into dialysis bags, which were placed in $200 \mathrm{~mL}$ of $\mathrm{PBS}(\mathrm{pH}=7.4)$. The release assay was performed at $37{ }^{\circ} \mathrm{C}$ with a stirring rate of $100 \mathrm{rpm}$. At given time intervals, $2 \mathrm{~mL}$ of solution were removed and $2 \mathrm{~mL}$ of fresh fluid were added immediately to keep the volume constant. The release of 5-HMF from the buffer solution was monitored at $298 \mathrm{~nm}$. 5-HMF@MSNs samples were used as a comparison and the release process remained the same as described above.

\section{Degradation of 5-HMF@MSNs}

A total of $5 \mathrm{mg}$ of 5-HMF@MSNs were evenly dispersed in $10 \mathrm{~mL}$ of PBS $(\mathrm{pH}=7.4)$, which was divided into six equal parts and

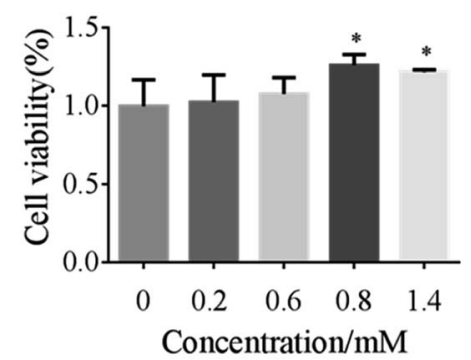

Fig. 3 The antioxidant effect of 5-HMF in UVB-exposed $\left(18 \mathrm{~mJ} \mathrm{~cm}{ }^{-2}\right)$ cells.
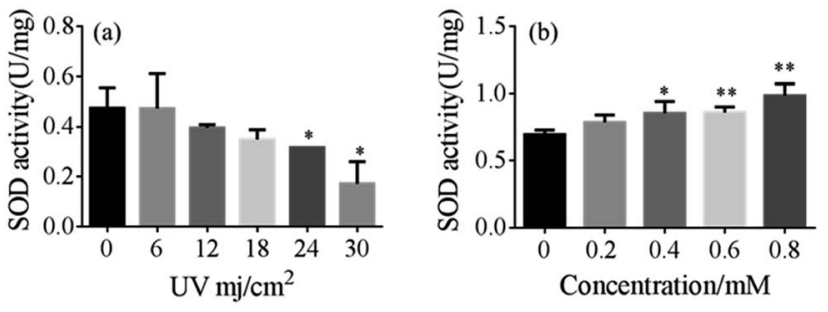

Fig. 4 (a) The effect of the UVB-exposed group on SOD activity and (b) and the effect of 5-HMF on SOD activity after UVB $\left(18 \mathrm{~mJ} \mathrm{~cm}^{-2}\right)$ exposure.

placed in a $2 \mathrm{~mL}$ centrifuge tube. These tubes were processed at $37{ }^{\circ} \mathrm{C}$ with a stirring rate of $100 \mathrm{rpm}$. The tube was withdrawn at 4 h, 12 h, 24 h, 48 h, 72 h, and 96 h, respectively.

\section{Cellular uptake}

MSNs $(0.5 \mathrm{~g})$ were dispersed in EtOH $(10 \mathrm{~mL})$, then hydrolyzed APTES $(200 \mu \mathrm{L})$ was injected into this dispersion with magnetic stirring $(500 \mathrm{rpm})$ at $70{ }^{\circ} \mathrm{C}$ for $12 \mathrm{~h}$. The AP-MSNs were collected
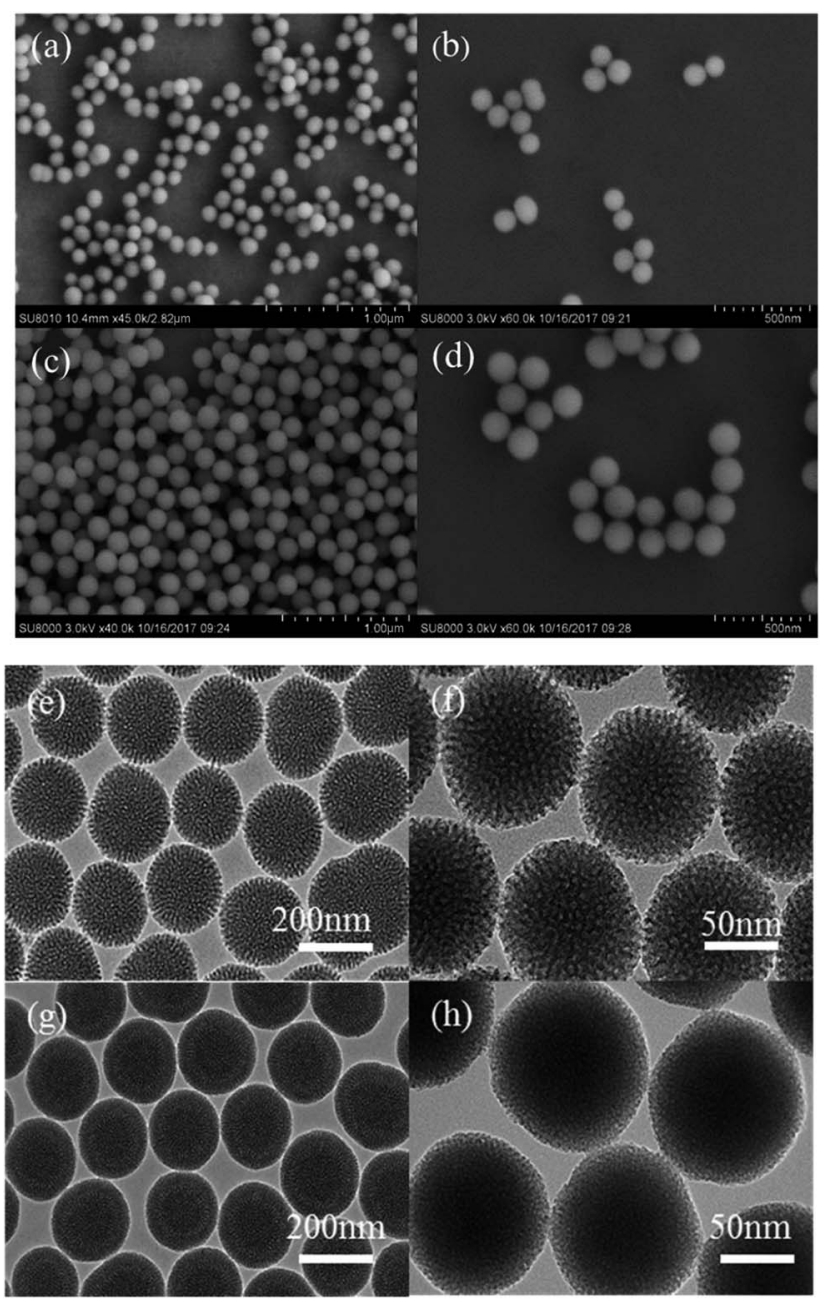

Fig. 5 (a) and (b), (e) and (f) are SEM and TEM images of 3D-dendritic MSNs, and (c) and (d), (g) and (h) are SEM and TEM images of 5HMF@MSNs. 


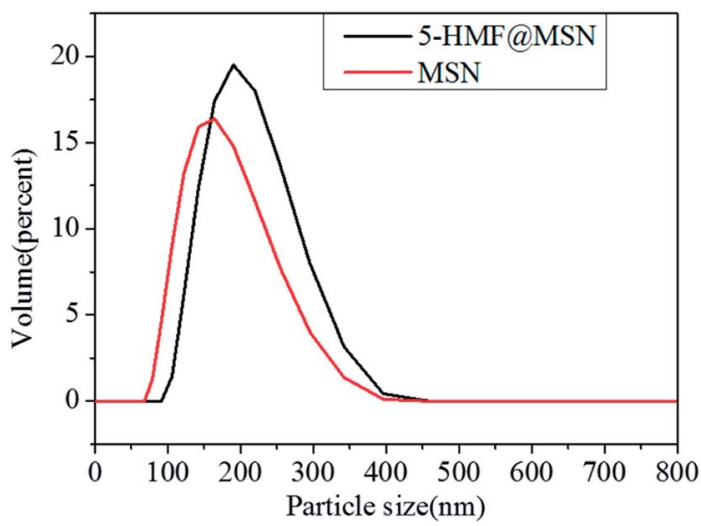

Fig. 6 Particle size distribution of MSN and 5-HMF@MSNs.

by centrifugation $(6500 \mathrm{rpm})$ for 10 minutes, washed three times with $\mathrm{EtOH}$, and dried under vacuum freeze. These products $(0.5 \mathrm{~g})$ were then dispersed in EtOH $(10 \mathrm{~mL})$ and injected into fluorescein isothiocyanate (FITC, $200 \mu \mathrm{L}$ ) with magnetic stirring $(500 \mathrm{rpm})$ at for room temperature for $4 \mathrm{~h}$.

To study the cellular uptake of 3D-dendritic MSNs qualitatively, HSF cells were seeded into cells climbing in the 24 -well plates at a density of $5 \times 10^{4}$ cells per $\mathrm{mL}$. The cells were allowed to attach in the growth medium at $37^{\circ} \mathrm{C}$ in a $5 \% \mathrm{CO}_{2}$ incubator for $24 \mathrm{~h}$. Next, the medium was removed and replaced with fresh medium containing FITC-labeled 3D-MSNs $\left(125 \mu \mathrm{g} \mathrm{mL}{ }^{-1}\right)$.
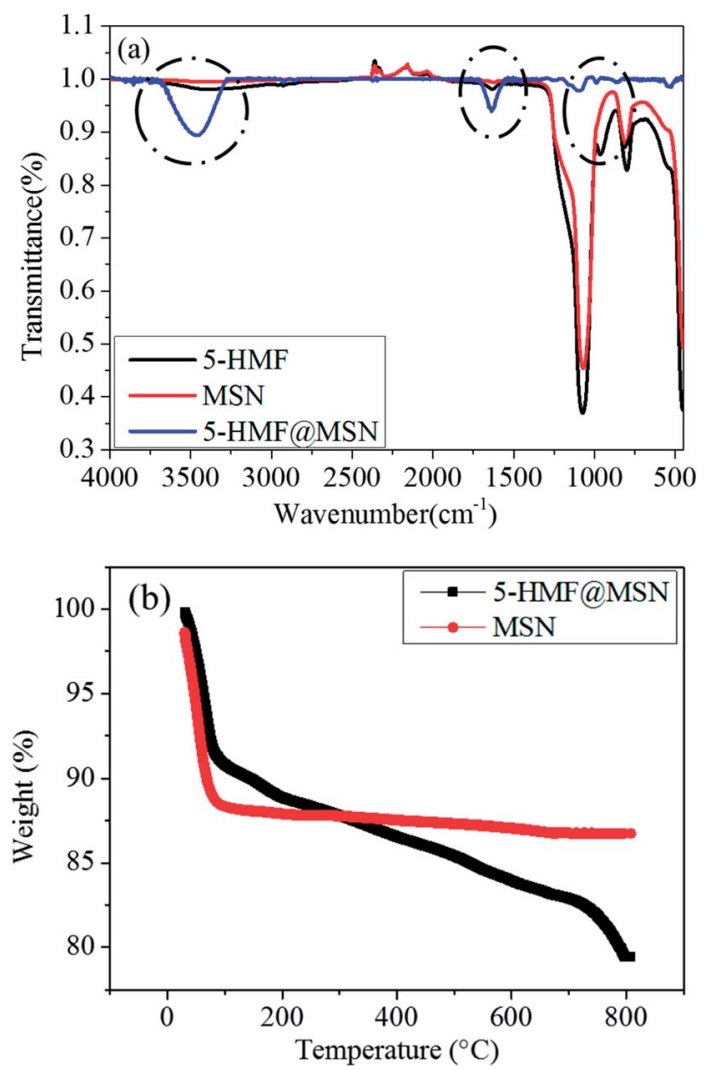

Fig. 7 (a) FT-IR spectra of pristine MSNs and 5-HMFaMSNs. (b) TGA curves of and MSNs, 5-HMFaMSNs.
After incubation of $1 \mathrm{~h}, 4 \mathrm{~h}, 24 \mathrm{~h}$, and $48 \mathrm{~h}$, the cells in each well were washed with PBS three times and $4 \%$ formaldehyde was added. The nuclei and cytomembrane were later stained by 4,6diamidino-2-phenylindole (DAPI) and wheat germ agglutinin (WGA) for $15 \mathrm{~min}$, respectively. Subsequently, cells were washed three times with PBS and observed by a confocal laser scanning microscope (A1, Nikon, Japan).

The quantitative cellular uptake study was performed using flow cytometry (FACS) analysis, and the cells were seeded into 12-well culture plates at a density of $10^{5}$ cells per mL. After $1 \mathrm{~h}$, $4 \mathrm{~h}, 24 \mathrm{~h}$, and $48 \mathrm{~h}$ of incubation with FITC-labeled 3D-MSNs $\left(125 \mu \mathrm{g} \mathrm{mL}{ }^{-1}\right)$, the cells were washed three times with PBS and trypsinized for $2 \mathrm{~min}$. Trypsinization was terminated by adding fresh medium. The cells were extracted gently by pipetting. After centrifugation, the treated samples were transferred to FACS tubes. The cells were analyzed by FACS (Beckman Coulter, USA).

\section{In vitro cytotoxicity assays}

The cytotoxicity of 3D-dendritic MSNs and 5-HMF@MSNs in various concentrations was investigated using the Thiazolyl Blue Tetrazolium Bromide (MTT) Cell Proliferation and Cytotoxicity Assay Kit. Briefly, HSF cells were seeded in 96-well plates with $100 \mu \mathrm{L}$ of medium at a density of $8 \times 10^{3}$ cells per well, and cultured in $5 \% \mathrm{CO}^{2}$ at $37^{\circ} \mathrm{C}$ for $24 \mathrm{~h}$. Various concentrations of 3D-MSNs and 5-HMF@MSNs $\left(25 \mu \mathrm{g} \mathrm{mL}{ }^{-1}, 31 \mu \mathrm{g} \mathrm{mL} L^{-1}, 62.5 \mu \mathrm{g}\right.$
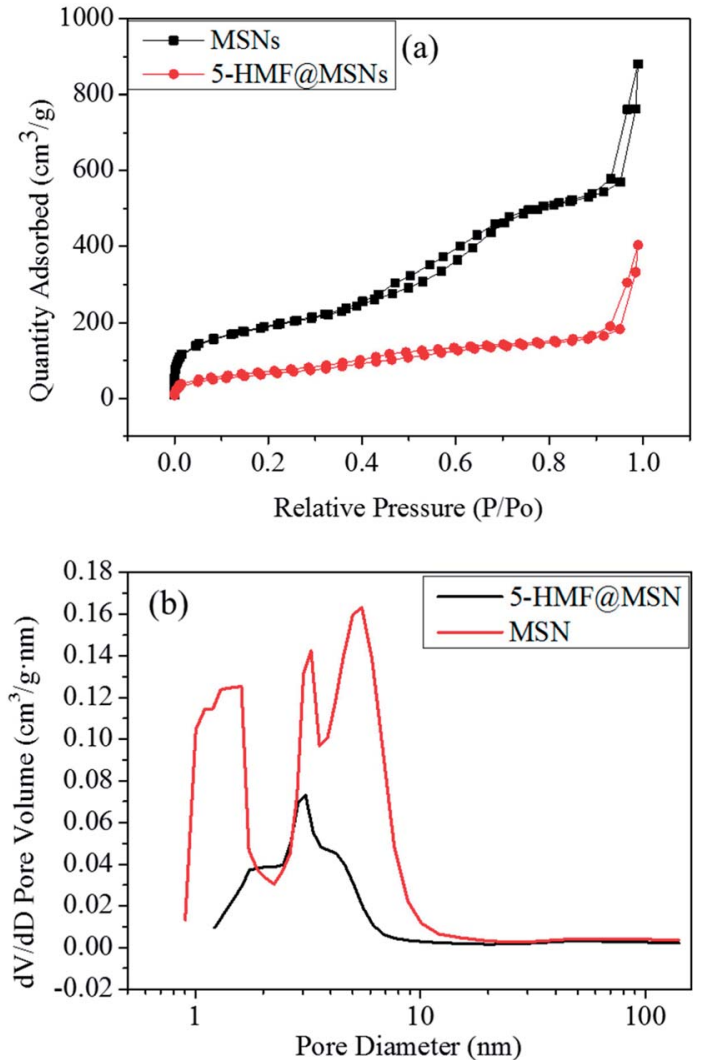

Fig. 8 (a) Nitrogen adsorption-desorption isotherms. (b) Pore size distribution. 
DAPI
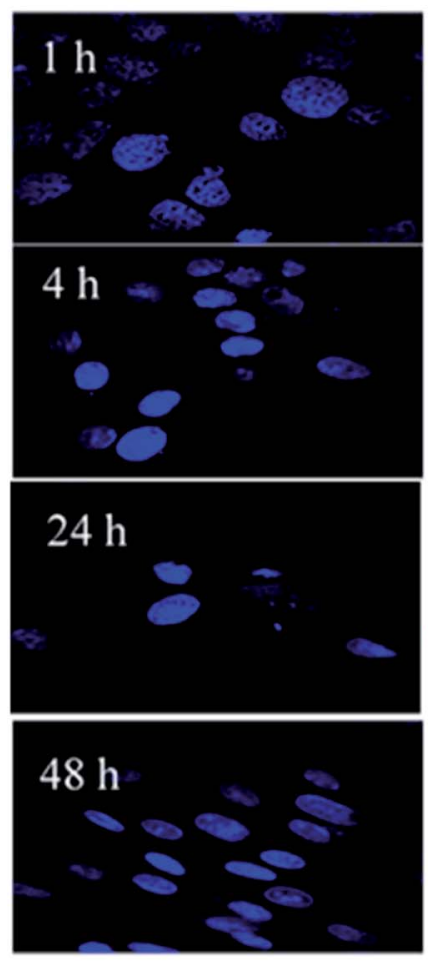

FITC
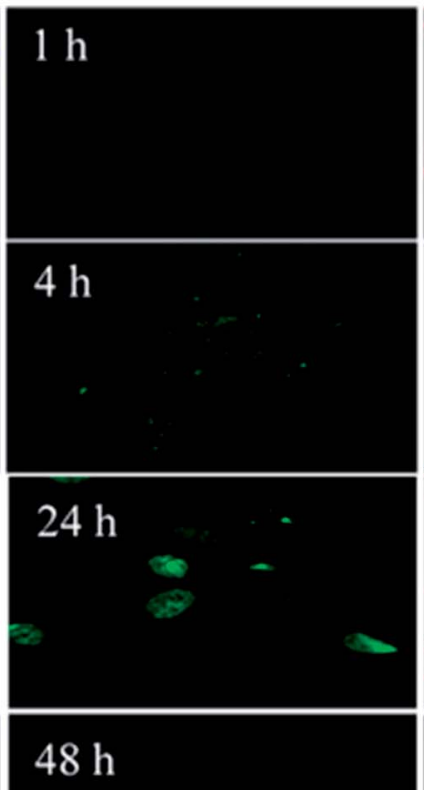

WGA
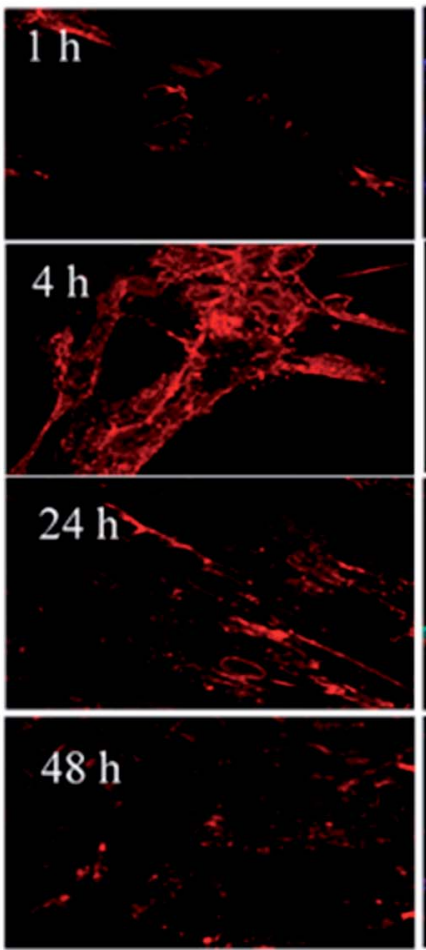

\section{MERGE}
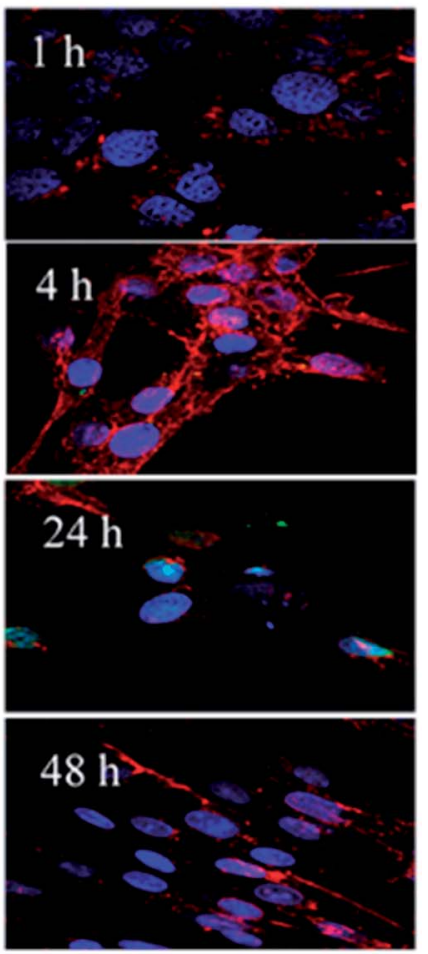

Fig. 9 Confocal microscopy images of HSF cells after treatment with FITC-labeled MSN for $1 \mathrm{~h}, 4 \mathrm{~h}, 24 \mathrm{~h}$, and $48 \mathrm{~h}$.

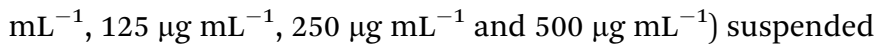
in culture medium were separately introduced into the cells.

After incubation for $24 \mathrm{~h}$, the medium was removed and 10 $\mu \mathrm{L}$ of MTT solution and $100 \mu \mathrm{L}$ fresh medium were added. The cells were incubated for another $4 \mathrm{~h}$ at $37^{\circ} \mathrm{C}$. Then the medium was removed again, $110 \mu \mathrm{L}$ of formazan solvent was added to the cell culture plate, and the cells were slowly oscillated on the shaker for $10 \mathrm{~min}$. The absorbance was measured at $570 \mathrm{~nm}$ with a microplate reader (Thermo Fisher, USA). A culture medium without nanospheres was used as the blank control. The cytotoxicity was expressed as the percentage of cell viability compared with the blank control.

\section{Antioxidant effect of 5-HMF@MSNs}

To assess the antioxidant properties of 5-HMF-loaded MSNs, the level of glutathione peroxide (GPx) was measured. In brief, various amounts of 5-HMF@MSNs were dispersed in fresh medium to obtain different concentrations $\left(75 \mu \mathrm{g} \mathrm{mL}{ }^{-1}, 125 \mu \mathrm{g}\right.$ $\left.\mathrm{mL}^{-1}, 250 \mu \mathrm{g} \mathrm{mL}{ }^{-1}, 500 \mu \mathrm{g} \mathrm{mL}^{-1}\right)$. HSF cells were seeded in 6well plates with $1000 \mu \mathrm{L}$ of medium at a density of $5 \times 10^{5}$ cells per well and allowed to attach in growth medium at $37^{\circ} \mathrm{C}$ in a $5 \% \mathrm{CO}_{2}$ incubator for $24 \mathrm{~h}$.

Then we removed the medium and added $100 \mu \mathrm{L}$ PBS. UVB irradiation was performed. Different concentrations of 5HMF@MSNs were added overnight. Experiments were performed according to the manufacturer's kit instructions as described above.

\section{Results and discussion}

The effect of UVB on the percentage of SA- $\beta$-gal-positive cells in UVB-SIPS fibroblasts

Compared with the no-radiation group, cells became irregular as the irradiation dose increased. The percentage of positive cells in the UVB-30 $\mathrm{mJ} \mathrm{cm} \mathrm{cm}^{-2}$ group was $78.43 \%$, whereas it was $0.4 \%$ in the control group. The percentage of positive cells in the UVB-6 mJ cm ${ }^{-2}$, UVB-12 $\mathrm{mJ} \mathrm{cm}{ }^{-2}$, UVB-18 $\mathrm{mJ} \mathrm{cm}^{-2}$, and UVB-24 $\mathrm{mJ} \mathrm{cm}{ }^{-2}$ groups were $1.3 \%, 19.54 \%, 27.77 \%$, and $65.45 \%$, respectively. A large number of cells died of UVB-30 mJ cm $\mathrm{m}^{-2}$ and UVB-24 mJ cm ${ }^{-2}$ exposure (Fig. 1e and f). UVB-6 $\mathrm{mJ} \mathrm{cm}^{-2}$

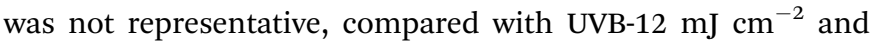
UVB-18 $\mathrm{mJ} \mathrm{cm}^{-2}$ as the optimal conditions for the establishment of the cell aging model. ${ }^{22}$ Fig. 2 shows the results of MTT at different doses.

\section{The cytotoxicity and antioxidant effects of 5-HMF}

Oxidative stress was one of the mechanisms of UVB-induced cell injury. ${ }^{23}$ We examined cell viability by MTT assays to evaluate the potential effect of 5-HMF against UVB-induced oxidative injury. We found that UVB treatment resulted in an obvious decrease in cell viability. By comparison, 5-HMF maintained cell survival at a relatively higher oxidative stress (Fig. 3).

These results suggested that 5-HMF played a protective role against oxidative injury. A number of enzymatic and nonenzymatic mechanisms have evolved to protect against ROS 
and free radicals resulting from oxidative stress in human skin fibroblast injury. ${ }^{24}$

SOD is one of the common antioxidant enzymes. As shown in Fig. 4 , the antioxidant SOD activities in the UVB- $18 \mathrm{~mJ} \mathrm{~cm}^{-2}$ group decreased by $12.52 \%$ with no 5-HMF. However, treatment with 5 -HMF for $24 \mathrm{~h}$ can effectively prevented the decrease.

\section{Characterization of 5-HMF@MSNs}

The morphologies and pore structures were characterized. The TEM and SEM images of 3D-dendritic MSNs and 5-HMF@MSNs are displayed in Fig. 5. From the SEM observation, drug loading had no impact on carrier shape.

Compared with the reference, we got a smaller diameter of MSNs by adjusting the pH. 3D-Dendritic MSNs had uniform spherical shapes with a diameter of $154.2 \mathrm{~nm}$ (Fig. 6) and good dispersion.

The charge of silica is determined by the $\mathrm{pH}$ of the synthesis solution. Under alkaline conditions, silicates with a high negative charge density are assembled with cationic surfactants through strong electrostatic interactions. In most of the synthetic processes for MSNs, when the $\mathrm{pH}$ is high $(\mathrm{pH}=9-13)$, the silicate dissolves in the aqueous solution. The silicate and the cationic surfactant pass violently between each other. ${ }^{21}$ As a result, the formed surfactant-silicate composite can be made stable. The $\mathrm{pH}$ of the reaction solution was adjusted to 10 with sodium hydroxide $(\mathrm{NaOH})$ through multiple tests, and we have obtained mesoporous silica with a very small particle size. Smaller particle size makes it easier for nanospheres to enter the cell and release drugs. ${ }^{25}$ After being loaded with 5-HMF, the size of 5-HMF@MSNs increased slightly (187.6 nm), which indicated the successful loading of 5-HMF molecules (Fig. 6). ${ }^{25}$

The force which make the hydrophilic drug (5-HMF) load into silica sphere may likely be hydrogen bond. It could be attributed to the $\mathrm{Si}-\mathrm{OH}$ groups in silica sphere which easily react with -OH groups in 5 -HMF. ${ }^{26}$ The same phenomenon has been reported by Q. Liu et al. ${ }^{27}$ They found that paclitaxel (PTX) and rapamycin (RAPA) were attracted through hydrogen bonding and polar interactions.

The LC\% and EE\% of drug delivery systems using different weight ratios of 5-HMF : MSNs $(0.5: 1,0.75: 1,1: 1,2: 1$, w/w $)$ were also calculated. The LC\% increased with increasing ratios of 5-HMF : MSNs, whereas the EE\% reached the highest value of about $52 \%$ when the initial weight of 5-HMF : MSNs was fixed at $0.5: 1$ and the loading of the 5 -HMF on silica sphere was $19 \%$.

To further support the successful loading of the 5-HMF drugs, the FTIR spectra of 3D-dendritic MSNs and 5HMF@MSNs are presented in Fig. 7a. The spectra of 5-HMF displayed three absorption peaks at $3473 \mathrm{~cm}^{-1}, 1629 \mathrm{~cm}^{-1}$, and $988 \mathrm{~cm}^{-1}$, which could be typical peaks of $-\mathrm{OH}$ groups (Fig. 7a, blue line). The spectra of 3D-dendritic MSNs displayed three absorption peaks at $1070 \mathrm{~cm}^{-1}, 805 \mathrm{~cm}^{-1}$, and $453 \mathrm{~cm}^{-1}$, which could be attributed to the bending vibration and stretching vibration of hydroxyl groups, respectively (Fig. 7a, red line). For the 5-HMF@MSNs, stronger peaks at $3525 \mathrm{~cm}^{-1}, 1648 \mathrm{~cm}^{-1}$, and $970 \mathrm{~cm}^{-1}$ were observed, which can be attributed to abundant $-\mathrm{OH}$ groups of 5 - $\mathrm{HMF}$ molecules, indicated the

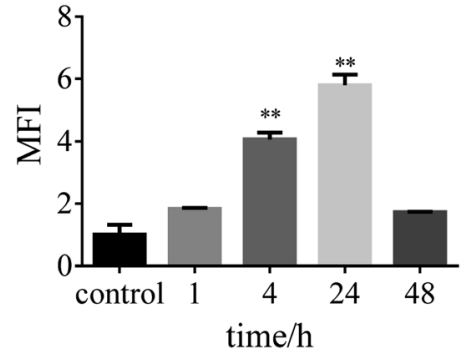

Fig. 10 Histogram result of flow cytometry.

successful immobilization of 5-HMF molecules inside the silicabased nanospheres (Fig. 7a, black line). ${ }^{28}$

The Thermogravimetric analysis (TGA) curves of 5HMF@MSNs and MSNs show two distinct weight losses (Fig. 7b). According to the data of the final sample residue, the loss rate of 5-HMF@MSNs was more than MSN. From $100{ }^{\circ} \mathrm{C}$ to $300{ }^{\circ} \mathrm{C}$, the temperature has perhaps not yet reached the temperature at which the chemical bond formed by the drug and the carrier is disconnected, so the loss of 5-HMF@MSNs was less than MSN. But there was still a trend of declining quality. The weight loss of 5-HMF@MSNs was $21 \%$ (Fig. 7b, black line) when heated in an $\mathrm{O}_{2}$ atmosphere to $800{ }^{\circ} \mathrm{C}$, whereas
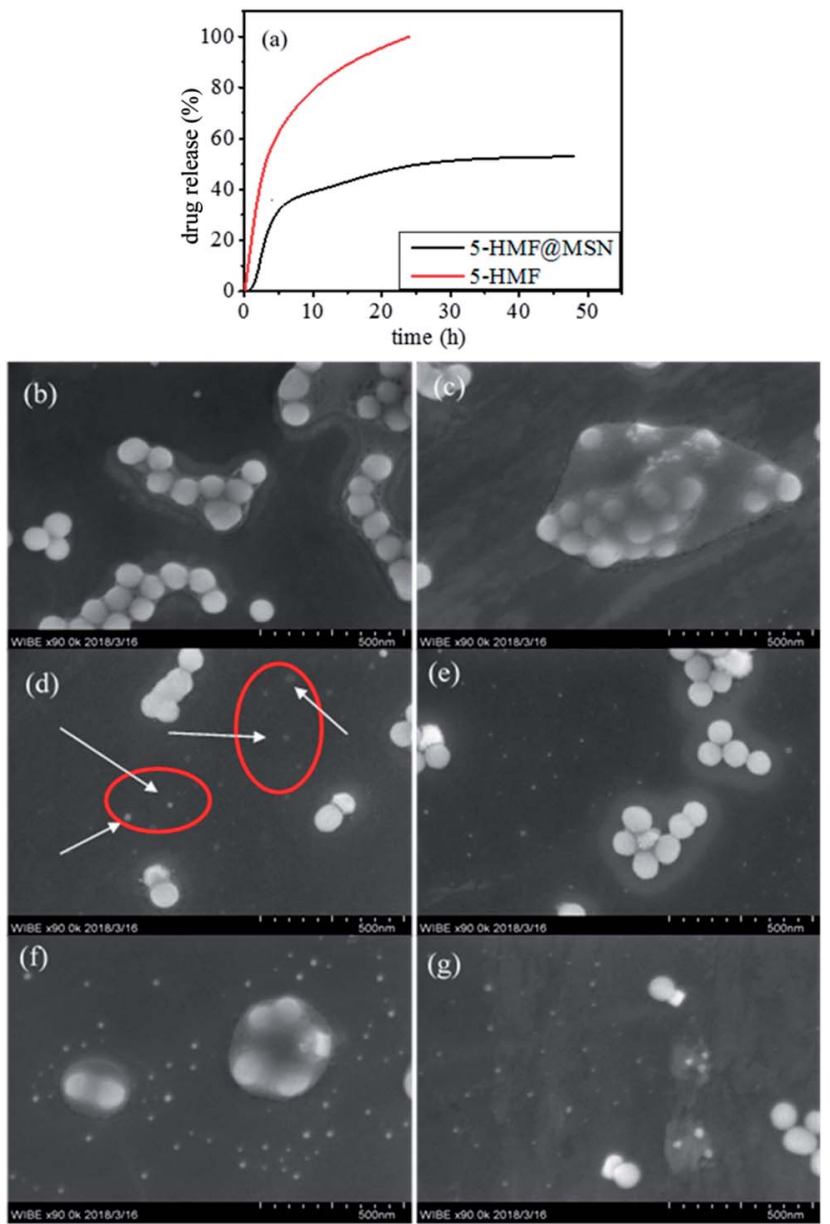

Fig. 11 (a) Cumulative release profiles of 5-HMF and 5-HMF@MSNs. $(\mathrm{b}-\mathrm{g})$ The SEM of degradative 5-HMF@MSNs. 
that of MSNs was only $12.5 \%$ (Fig. $7 \mathrm{~b}$, red line) in the same temperature range. The results of TGA further demonstrated that 5-HMF was successfully loaded on MSNs. ${ }^{29,30}$

We can see in Fig. 9 that the surface areas $\left(S_{\text {bet }}\right)$ and cumulative pore volumes $\left(V_{\mathrm{t}}\right)$ of MSNs were $663.6667 \mathrm{~m}^{2} \mathrm{~g}^{-1}$ and 1.35 $\mathrm{cm}^{3} \mathrm{~g}^{-1}$. After the drug loading and grafting process, the $S_{\text {bet }}$ and $V_{\mathrm{t}}$ of 5-HMF@MSNs decreased significantly, which can be ascribed to the successful loading of the 5-HMF molecules. The capillary condensation step expands to $0.2<P / P_{0}<0.7$ in the $\mathrm{N}_{2}$ sorption isotherms, and the hysteresis loops appear in $0.4<P / P_{0}$ $<0.7$ (Fig. 9a, black line), implying a bimodal mesopore. The pore size distribution was analyzed using the $\mathrm{BJH}$ method applied to the adsorption branch of the isotherm, a micropore of $\sim 1.5 \mathrm{~nm}$ for the first generation, a middle mesopore of $\sim 3.2 \mathrm{~nm}$ for the second generation, and a large pore of $\sim 5.5 \mathrm{~nm}$ for the third generation (Fig. 8b, red line). The 5-HMF pore size was obviously smaller than the 5-HMF@MSNs pore size, (Fig. 8b, black line). ${ }^{31}$

\section{Cellular uptake}

The fluorescent confocalization of cellular uptake by nanocarriers is shown in Fig. 9. At $4 \mathrm{~h}$, it can already be seen that there are carriers in the cells. Within $24 \mathrm{~h}$, the nanocarriers completely entered the cell. The result of flow cytometry coincides with the result of fluorescence confocalization (Fig. 10).
The fluorescence gradually disappears after $48 \mathrm{~h}$. The condition may be attributed to carrier degradation or fluorescence quenching. ${ }^{32,33}$

\section{In vitro drug release properties}

Drug was gradually released through degradation of the carrier in vivo. From the SEM results, carrier degradation is faster than traditional silicon balls, which reduced the accumulation of carriers, thereby alleviating damage to the human liver and spleen. ${ }^{13}$ To evaluate the drug release properties of the 5HMF@MSNs and 5-HMF, drug-loaded samples were dispersed in different PBS solutions $(\mathrm{pH}=7.4) .^{33}$

As shown in Fig. 11a, the cumulative release rate of 5-HMF without carrier loading reached about $60 \%$ in $4 \mathrm{~h}$ and increased to $99 \%$ in $24 \mathrm{~h}$. As a three-dimensional mesoporous silica carrier, the pores were large and the wall was thin in the outermost dimension, so the degradation was relatively easy. Therefore, the initial drug release of 5-HMF@MSNs was caused by the degradation of the outermost layer. The drug release rate was maintained when the drug concentration was kept constant. The cumulative release rate of 5-HMF@MSNs in PBS $(\mathrm{pH}=7.4)$ increased to $55 \%$ within $48 \mathrm{~h}$. SEM images at $4 \mathrm{~h}$, 12 h, 24 h, 48 h, 72 h, and 96 h (Fig. 11b-g) show the degradation of 5-HMF@MSNs. It can be clearly observed that the nanospheres gradually degrade over time (Fig. 11d).
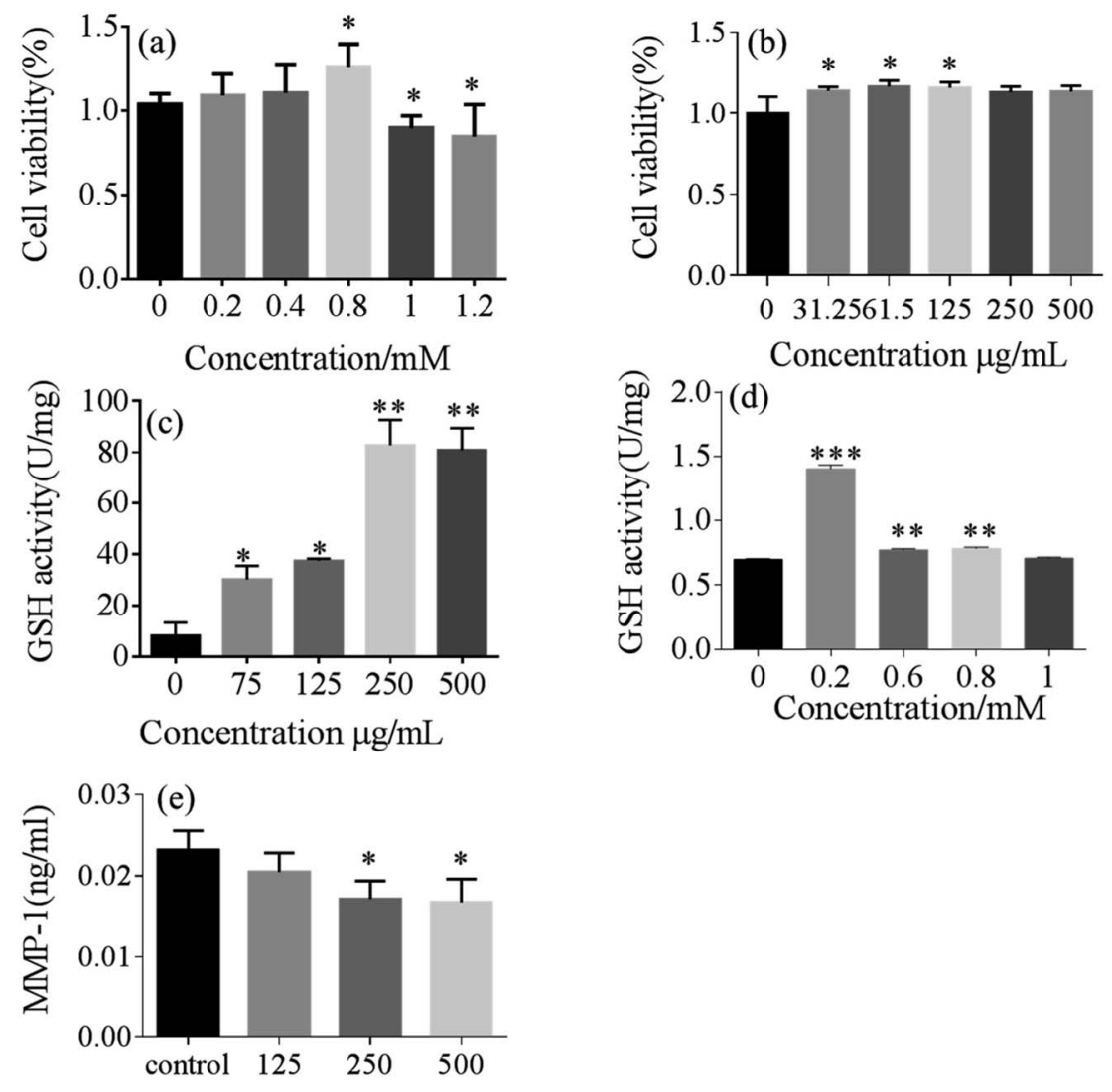

Concentration $\mu \mathrm{g} / \mathrm{mL}$

Fig. 12 The multiplication of 5-HMF (a) and 5-HMF@MSNs (b). Activity of GPx for 5-HMF (c) and 5-HMFaMSNs (d) and the effect of 5-HMF on MMP-1 production (e) in UVB-irradiated HSF $\left(18 \mathrm{~mJ} \mathrm{~cm}^{-2}\right)$. 


\section{In vitro biocompatibility evaluation and antioxidant properties}

To evaluate the biocompatibility of the silica-based nanosystem, HSF cells were incubated with bare 5-HMF and 5-HMF@MSNs to assess the cytotoxicity using MTT assays. As depicted in Fig. 12, the 5-HMF@MSNs were biocompatible. At similar doses, the biological toxicity of 5-HMF@MSNs was lower than that of bare 5-HMF due to the slow release of the drug in the carriers.

For the purpose of investigating the antioxidant properties of 5-HMF@MSNs and pure 5-HMF, GPx assays were carried out. $^{34,35}$ Typically, a range of gradient concentrations of 5HMF@ MSNs and pure 5-HMF were added to the induced cells. The GPx activity of 5-HMF@MSNs was higher than that of pure 5-HMF when the doses of 5-HMF were similar.

The effect of 5-HMF@MSNs on MMP-1 production in fibroblasts exposed to UVB radiation at an exposure dose of $18 \mathrm{~mJ}$ $\mathrm{cm}^{-2}$ was shown in Fig. 12. ${ }^{36}$ UVB radiation significantly increased matrix metalloproteinase (MMP-1) production in HSF. However, the pretreatment of fibroblasts with 5HMF@MSNs of $250 \mu \mathrm{g} \mathrm{mL} \mathrm{m}^{-1}$ and $500 \mu \mathrm{g} \mathrm{mL}^{-1}$ reduced the MMP-1 production by $26.7 \%$ and $28 \%$, respectively. The secretion of MMP-1 significantly decreased in a dose-dependent manner in this UVB dermal damage model.

\section{Conclusions}

Currently, MSNs are often used as a transport carrier for hydrophobic macromolecular drugs. ${ }^{25}$ However, their use in preventing ultraviolet damage is documented.

Nanometer carriers can protect the physico-chemical and biological properties of active ingredients. ${ }^{37}$ In this study, we developed a novel strategy to fabricate a type of 3D-dendritic mesoporous silica framework for delivery of the small molecular hydrophilic drug 5-HMF. The nanocarriers can effectively enhance the bioavailability and antioxidant properties of the drugs. Moreover, slow drug release was observed after the introduction of the nanocarrier, which could reduce drug toxicity. ${ }^{38}$

In addition, in vitro cell experiments were conducted to confirm the excellent biocompatibility and low cytotoxicity of the well-designed nanocarriers. Therefore, this novel nanosystem has great potential application in the future development of water-soluble and active drug delivery and in the cosmetics industry.

\section{Conflicts of interest}

The author declares no conflict of interest.

\section{Acknowledgements}

This research was financially supported by the International Cooperation Projects of Guangdong Provincial Science and Technology (Grant No. 2015A050502013), the National Natural Science Foundation of China (21707060), and Multi-stage construction of high-strength microsphere scaffolds and development of bone repair materials (Grant No. 2015A020214005).

\section{Notes and references}

1 M. Cavinato and P. Jansen-Durr, Exp. Gerontol., 2017, 94, 7882.

2 E. D. Lephart, Ageing Res. Rev., 2016, 31, 36-54.

3 C. C. Li, Y. T. Lin, Y. T. Chen, S. F. Sie and Y. W. Chen-Yang, J. Photochem. Photobiol., B, 2015, 148, 277-283.

4 S. B. Nimse and D. Pal, RSC Adv., 2015, 5, 27986-28006.

5 E. Sayed, R. Haj-Ahmad, K. Ruparelia, M. S. Arshad, M. W. Chang and Z. Ahmad, AAPS PharmSciTech, 2017, 18, 1507-1525.

6 M. Diaz, H. Degens, L. Vanhees, C. Austin and M. Azzawi, Exp. Gerontol., 2016, 85, 41-47.

7 L. Zhao, J. Chen, J. Su, L. Li, S. Hu, B. Li, X. Zhang, Z. Xu and T. Chen, J. Agric. Food Chem., 2013, 61, 10604-10611.

8 A. Liu, X. Zhao, H. Li, Z. Liu, B. Liu, X. Mao, L. Guo, K. Bi and Y. Jia, Int. Immunopharmacol., 2014, 23, 719-725.

9 H. K. Kim, Y. W. Choi, E. N. Lee, J. K. Park, S. G. Kim, D. J. Park, B. S. Kim, Y. T. Lim and S. Yoon, Phytother. Res., 2011, 25, 965-974.

10 Y. X. Li, Y. Li, Z. J. Qian, M. M. Kim and S. K. Kim, J. Microbiol. Biotechnol., 2009, 19, 1319-1327.

11 D. Patra, D. Şen Karaman, D. Desai, E. El Khoury and J. M. Rosenholm, Mater. Res. Bull., 2016, 84, 267-272.

12 A. Bagde, A. Mondal and M. Singh, Photodermatol., Photoimmunol. Photomed., 2018, 34, 60-68.

13 D. Shen, J. Yang, X. Li, L. Zhou, R. Zhang, W. Li, L. Chen, R. Wang, F. Zhang and D. Zhao, Nano Lett., 2014, 14, 923932.

14 D. Shen, L. Chen, J. Yang, R. Zhang, Y. Wei, X. Li, W. Li, Z. Sun, H. Zhu, A. M. Abdullah, A. Al-Enizi, A. A. Elzatahry, F. Zhang and D. Zhao, ACS Appl. Mater. Interfaces, 2015, 7, 17450-17459.

15 X. L. Xue, W. Z. Lang, X. Yan and Y. J. Guo, ACS Appl. Mater. Interfaces, 2017, 9, 15408-15423.

16 L. Xu, D. Wu, B. Zhou, Y. Xu, W. Wang, D. Yu and D. Luo, RSC Adv., 2018, 8, 12315-12321.

17 S. Mura, J. Nicolas and P. Couvreur, Nat. Mater., 2013, 12, 991-1003.

18 S. Iraji, L. Rashidi and F. Ganji, Iranian Journal of Chemical Engineering, 2015, 12, 93-100.

19 Y. Zhu and C. Tao, RSC Adv., 2015, 5, 22365-22372.

20 M. Martínez-Carmona, D. Lozano, M. Colillaa and M. ValletRegí, RSC Adv., 2016, 6, 50923-50932.

21 S.-H. Wu, C.-Y. Mou and H.-P. Lin, Chem. Soc. Rev., 2013, 42, 3862-3875.

22 A. Bowman and M. A. Birch-Machin, J. Invest. Dermatol., 2016, 136, 912-919.

23 K. Bravo, L. Duque, F. Ferreres, D. A. Moreno and E. Osorio, J. Photochem. Photobiol., B, 2017, 168, 78-88.

24 M. Cavinato, R. Koziel, N. Romani, R. Weinmullner, B. Jenewein, M. Hermann, S. Dubrac, G. Ratzinger, J. Grillari, M. Schmuth and P. Jansen-Durr, J. Gerontol., Ser. A, 2017, 72, 632-639. 
25 Z. Mai, J. Chen, Y. Hu, F. Liu, B. Fu, H. Zhang, X. Dong, W. Huang and W. Zhou, J. Colloid Interface Sci., 2017, 508, 184-195.

26 J. M. Rosenholm, C. Sahlgren and M. Linden, Nanoscale, 2010, 2, 1870-1883.

27 Q. Liu, J. Zhang, W. Sun, Q. R. Xie, W. Xia and H. Gu, Int. J. Nanomed., 2012, 7, 999-1013.

28 S. Sapino, E. Ugazio, L. Gastaldi, I. Miletto, G. Berlier, D. Zonari and S. Oliaro-Bosso, Eur. J. Pharm. Biopharm., 2015, 89, 116-125.

29 A. Hakeem, F. Zahid, R. Duan, M. Asif, T. Zhang, Z. Zhang, Y. Cheng, X. Lou and F. Xia, Nanoscale, 2016, 8, 5089-5097.

30 D. Chang, Y. Gao, L. Wang, G. Liu, Y. Chen, T. Wang, W. Tao, L. Mei, L. Huang and X. Zeng, J. Colloid Interface Sci., 2016, 463, 279-287.

31 G. Berlier, L. Gastaldi, S. Sapino, I. Miletto, E. Bottinelli, D. Chirio and E. Ugazio, Int. J. Pharm., 2013, 457, 177-186.

32 N. Zheng, J. Li, C. Xu, L. Xu, S. Li and L. Xu, Artif. Cells, Nanomed., Biotechnol., 2017, 1-9.
33 J. Chen, N. Wei, M. Lopez-Garcia, D. Ambrose, J. Lee, C. Annelin and T. Peterson, Eur. J. Pharm. Biopharm., 2017, 117, 286-291.

34 Y. Yang and S. Li, Oxid. Med. Cell. Longevity, 2015, 2015, 110.

35 G. Valacchi, C. Sticozzi, A. Pecorelli, F. Cervellati, C. Cervellati and E. Maioli, Ann. N. Y. Acad. Sci., 2012, 1271, 75-81.

36 E. Carazo, A. Borrego-Sanchez, F. Garcia-Villen, R. SanchezEspejo, P. Cerezo, C. Aguzzi and C. Viseras, Chem. Rec., 2018, 18, 1-108.

37 E. Ugazio, L. Gastaldi, V. Brunella, D. Scalarone, S. A. Jadhav, S. Oliaro-Bosso, D. Zonari, G. Berlier, I. Miletto and S. Sapino, Int. J. Pharm., 2016, 511, 446-454.

38 E. Sayed, R. Haj-Ahmad, K. Ruparelia, M. S. Arshad, M. W. Chang and Z. Ahmad, AAPS PharmSciTech, 2017, 18, 1507-1525. 